\title{
Tracking control of cascade systems based on passivity: The non- adaptive and adaptive cases
}

\author{
Juan C. Travieso-Torres, Manuel A. Duarte-Mermoud, ${ }^{*}$ Jorge L. Estrada \\ Department of Electrical Engineering, University of Chile, Av. Tupper 2007, Casilla 412-3, Santiago, Chile
}

(Received 19 April 2005; accepted 11 December 2005)

\begin{abstract}
A new cascade passivity-based control scheme for tracking purposes is proposed in this paper. The proposed scheme is valid for a certain class of nonlinear systems even with unstable zero dynamic, and it is also useful for regulation and stabilization purposes. The cases where all system parameters are assumed to be known (nonadaptive case) and also the case when they are unknown (adaptive case) are considered. Some simulation examples are studied to analyze the behavior of the proposed scheme. (C) 2006 ISA-The Instrumentation, Systems, and Automation Society.
\end{abstract}

Keywords: Passivity-based control (PBC); Adaptive passivity; Adaptive tracking; Adaptive regulation; Adaptive stabilization; Adaptive cascade control

\section{Introduction}

Passivity-based control (PBC) is an important methodology for feedback stabilization of nonlinear systems [1] that has been widely studied in the last three decades [2-5]. The main advantage of the PBC over other nonlinear control techniques is that the control is designed taking into account the energy of the system and then variables have a clear physical meaning. Besides, this technique is less complex than backstepping control algorithms, which require several steps to design the controller, or linearization techniques where the diffeomorphism is difficult to define and knowledge of the plant parameters is involved.

A synthesis of concepts and conditions for nonadaptive PBC are presented in [5], where the proposed scheme is valid for stabilization purposes, and assumes all system parameters to be known.

\footnotetext{
*Author to whom all correspondence should be addressed. E-mail address: mduartem@cec.uchile.cl
}

Later, adaptive PBC was proposed in [6-9]. The techniques proposed there are also applicable for stabilization but assume all system parameters to be unknown.

Based on the equivalence between model reference adaptive control (MRAC) [10] and adaptive PBC established in [11], a generalized PBC scheme for tracking purposes was proposed very recently in [12].

In the context of cascade systems, work has been done in order to determine the passivity properties for stabilization of cascade nonlinear system [13] for the case when the plant parameters are known. Recently, an $L^{\infty}$ approach has been proposed in [14] for bounded robust control of nonlinear cascade systems with disturbances.

The results presented in this paper are based on the previous work presented in [5-9] and it considers a certain class of nonlinear system in cascade, with a known structure and known state variables. The results obtained constitute a new control scheme for tracking purposes that considers the nonadaptive case, where all the system pa- 
rameters are assumed to be known, but also considers the adaptive case to study the case when all system parameters are unknown. The methodology is applicable to systems with unstable zero dynamics, so that the assumption that the system is locally nonminimum phase is not needed.

Compared with the conventional PID industrial controller designed for regulation purposes, used for instance in CA and CC electrical motor drives to control speed and torque, the adaptive $\mathrm{PBC}$ scheme proposed here uses a simple proportional controller. Therefore, a much simpler tuning and a more robust and better behavior for tracking applications is expected with this scheme if used instead of a PID controller.

The paper is organized as follows. The concepts of nonadaptive and adaptive PBC for stabilization of nonlinear systems are briefly addressed in Section 2. In Section 3, a general cascade PBC scheme for the nonadaptive and adaptive cases is proposed. Two examples are studied by simulation and the results are presented in Section 5. Finally, in Section 5, some conclusions are drawn.

\section{PBC related concepts}

Let us consider a nonlinear system of the form

$$
\begin{gathered}
\dot{x}(t)=f(x)+g(x) u(t), \\
y(t)=h(x)
\end{gathered}
$$

with state space $X=R^{n}$, a set of input values $U$ $=R^{m}$, and a set of output values $Y=R^{m}$. The set $\mathcal{U}$ of admissible inputs consists of all piecewise continuous functions $R \rightarrow U=R^{m}$. Besides, $f \in R^{n}$, the $m$ columns of $g \in R^{n \times m}$ and $h \in R^{m}$ are smooth vector fields (i.e., $f, g, h \in C^{\infty}$ ). It is supposed that the vector field $f$ has at least one equilibrium point, and without loss of generality, after possibly a coordinates shift, it is possible to write that $f(0)=0$ and $h(0)=0$.

Next, for completeness we recall some definitions and concepts from [5], for the particular case of systems of the form (2.1).

Definition 2.1 [5]: For the system (2.1), if the matrix $L_{g} h(x)=[\partial h(x) / \partial x] g(x)$ is nonsingular in a neighborhood of $x=0$, then system (2.1) has a socalled relative degree $\{1,1,1, \ldots, 1\}$ at $x=0 . \diamond$

It is shown in [5] that if system (2.1) has relative degree $\{1,1, \ldots, 1\}$ at $x=0$ and the distribution spanned by vector fields $g_{1}(x), \ldots, g_{m}(x)$ is involutive [15], then it is possible to find new set of local coordinates $z(x) \in R^{n-m}, \quad m \leqslant n$, defined around $x=0$ and vanishing at $x=0$, under which this system, together with the $m$ components of the output map $y=h(x)$, can be represented in the normal form as follows:

$$
\begin{gathered}
\dot{y}(t)=a(A, y, z)+b(B, y, z) u(t), \\
\dot{z}(t)=c(y, z),
\end{gathered}
$$

where $a(A, y, z) \in R^{m}, b(B, y, z) \in R^{m \times m}, c(y, z)$ $\in R^{n-m}$, and $b(B, y, z)$ is invertible for all $(y, z)$ around $(0,0)$. $A, B$, and $C$ are a symbolic representation of the system parameters.

The zero dynamics of the system (2.2), denoted as $c(0, z)=f_{0}(C, z) \in R^{n-m}$, is defined [5] as those internal dynamics which are consistent with the external constraint $y=0$. Thus, the system (2.2) can be represented as

$$
\begin{gathered}
\dot{y}(t)=a(A, y, z)+b(B, y, z) u(t), \\
\dot{z}(t)=f_{0}(C, z)+p(D, y, z) y(t),
\end{gathered}
$$

where $p(D, y, z) \in R^{(n-m) \times m}$ is a smooth function. Note that $c(y, z)=f_{0}(C, z)+p(D, y, z) y \in R^{n-m}$.

A system whose zero dynamics are asymptotically stable is called minimum phase system.

Definition 2.2 [5]: Let us assume that $L_{g} h(0)$ is nonsingular. The system (2.1) is said to be locally weakly minimum phase if there exists a positive definite $C^{r}$ function $W_{0}(z)$, locally defined near 0 , with $r \geqslant 2$ and $W_{0}(0)=0$, satisfying

$$
L_{f_{0}(z)} W_{0}(z)=\frac{\partial W_{0}(x)}{\partial z} f_{0}(z) \leqslant 0
$$

for all $z$ in the neighborhood of $z(t)=0$.

Definition 2.3 [5]: A system of the form (2.1) is said to be $C^{r}$-passive if there exists a $C^{r}$ nonnegative function $V: R^{n} \rightarrow R$, called storage function, with $V(0)=0$, such that for all $u \in \mathcal{U}$, for all $x(0)=x_{0} \in X$ and $t \geqslant 0$, it is satisfied,

$$
V(x(t))-V\left(x_{0}\right) \leqslant \int_{0}^{t} y^{T}(s) u(s) d s .
$$

$V$ is a continuous storage function with continuous $r$-order derivatives $\left(V \in C^{r}\right)$. Condition (2.5) can also be expressed as $\dot{V} \leqslant y(t)^{T} u(t)$.

The system is said to be lossless for the case when $\dot{V}=y(t)^{T} u(t)$.

Passivity is a property that some physical systems possess and it is related to the input-output 
stability concept. A dynamical system is passive if the amount of energy stored by the system is lesser than the energy supplied to the system, i.e., the system internally does not generate energy but it dissipates some energy. Typical examples of practical passive systems are electrical circuits with passive elements (resistances, inductances, and capacitors) [15], an induction motor [16], etc.

Definition 2.4 [5]: A system of the form (2.1) is locally zero-state detectable if there exists a neighborhood $N$ of 0 such that for all $x_{0} \in N$ we have

$$
\begin{aligned}
h\left(\Phi\left(t, x_{0}, 0\right)\right) & =0 \quad \text { for all } t>0 \Rightarrow \lim _{t \rightarrow \infty} \Phi\left(t, x_{0}, 0\right) \\
& =0,
\end{aligned}
$$

where $\Phi\left(t, x_{0}, 0\right)$ denotes the state response at time $t$ for zero input $u$, starting at the initial state $x_{o}$ at $t=0$. If $N=X$, then the system is said to be zero-state detectable.

Definition 2.5 [5]: The system (2.1) is said to be locally equivalent via feedback to a $C^{r}$-passive system if there exists a state feedback $u(t)$ such that the resulting closed-loop system is passive with a $C^{r}$-storage function $V$.

The conditions under which a system is locally equivalent via feedback to a passive system are given in [5]. They are relative degree $\{1,1,1, \ldots, 1\}$ and locally weakly minimum phase.

Theorem 2.1 [5]: Let us consider a passive system of the form (2.1) with a $C^{r}(r \geqslant 1)$ storage function $V$, which is positive definite. Suppose the system is locally zero-state detectable. Then the control law

$$
u(t)=-K y(t),
$$

with $K \in \mathfrak{R}^{m \times m}$ a positive definite matrix, asymptotically stabilizes the system around the equilibrium point $x=0$. Moreover, if $V$ is proper, then the control law (2.7) globally asymptotically stabilizes the equilibrium $x=0$.

For a system of the form (2.2), being locally weakly minimum phase, the PBC proposed in [5] for stabilization purposes is shown in Fig. 1. This scheme is discussed in detail in Section 2.1.

This control scheme considers the application of a feedback to the system in order to obtain a passive equivalent system between the new input $u^{p}(t)$ and the output $y(t)$, and then uses the results of Theorem 2.1, which uses a simple proportional controller. In this paper, to simplify the exposition, a proportional controller will be used throughout

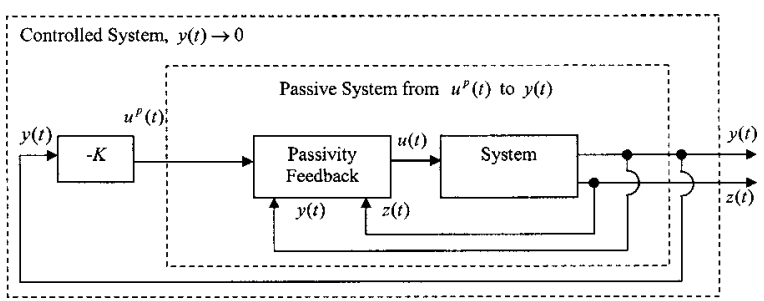

Fig. 1. PBC scheme for stabilization.

all the developments. However, a more complex controller of the form $u^{p}(t)=-\varphi(y)$, with $\varphi^{T}(y) y$ $>0$ and $\varphi(0)=0$ (similar to that used in [5]), can also be used and all the results presented here are still valid.

This type of strategy, in which a nonpassive system (or even a passive system but with some undesired characteristics) is made passive by a feedback, is quite common and has been used in many types of practical systems such as position control of levitation systems $[17,18]$ and speed and torque control of induction motors [19]. Another example where this kind of technique can be successfully used is in the control of a tank-truck half full of liquid. When the truck enters in a highway curve at high speed, we will have an undesired out of control behavior because of the liquid wave inside the tank, which is considered an active load. In order to control the tank vertical position, the system can be first turned passive via a state feedback and the controlled.

\subsection{Non-adaptive and adaptive passivity feedback}

We will first assume that all plant parameters are known.

Definition 2.6 [5]: The system (2.2) is said to be $C^{r}$-passive equivalent via feedback to a lossless system if there exists a state feedback of the form

$$
\begin{gathered}
u(t)=b(B, y, z)^{-1} \theta^{T} \omega(t), \\
\theta^{T}=\left[-I_{m} I_{m}\right], \quad \omega(t)=\left[a^{T}(A, y, z) u^{p T}(t)\right]^{T},
\end{gathered}
$$

where $a(A, y, z) \in \mathfrak{R}^{m}$ and $b(B, y, z) \in \mathfrak{R}^{m \times m}$ are smooth functions defined either locally near $x=0$ or globally and $b(B, y, z)$ is invertible for all $y(t), z(t)$, such that for the closed-loop system the following is satisfied: 


$$
\begin{aligned}
\dot{V}(y)= & \left(\frac{\partial V(y)}{\partial y}\right)^{T}\left\{a(A, y, z)+b(B, y, z) b(B, y, z)^{-1}\right. \\
& \left.\times\left[-a(A, y, z)+u^{p}(t)\right]\right\}=y(t)^{T} u^{p}(t), \quad(2.9
\end{aligned}
$$

where $V$ is a $C^{r}$-storage function and $u^{p}(t) \in \mathfrak{R}^{m}$ is the new control input.

Adaptive feedback passivity can be studied for a class of nonlinear system of the form (2.2) with linear explicit parametric dependence of the form

$$
\begin{gathered}
\dot{y}(t)=A a^{\prime}(y, z)+B b^{\prime}(y, z) u(t), \\
\dot{z}(t)=c(y, z),
\end{gathered}
$$

where $a^{\prime}(y, z) \in \mathfrak{R}^{m}, A \in \mathfrak{R}^{m \times m}, b^{\prime}(y, z) \in \mathfrak{R}^{m \times m}$, $B \in \mathfrak{R}^{m \times m}$ diagonal, $c(y, z) \in \mathfrak{R}^{n-m}$, and $b^{\prime}(y, z)$ is invertible for all $(y, z)$ around $(0,0)[6,7]$. For the system (2.10), we use the following adaptive passivity control law.

Definition 2.7 [6,7]: The system (2.10) is said to be $C^{r}$-passive equivalent via feedback to a lossless system, if there exists a state feedback of the form

$$
\begin{gathered}
u(t)=b^{\prime}(y, z)^{-1} \theta(t)^{T} \omega(t), \\
\theta^{T}(t)=\left[\begin{array}{ll}
\theta_{1}(t) & \left.\theta_{2}(t)\right] \in \mathfrak{R}^{m \times 2 m}, \\
\omega(t)=\left[a^{\prime T}(y, z)\right. & u^{p T}(t)
\end{array}\right]^{T} \in \mathfrak{R}^{2 m}
\end{gathered}
$$

with

$$
\dot{\theta}(t)^{T}=-\operatorname{sgn}(B) y(t) \omega(t)^{T},
$$

where $\theta_{1}(t) \in \mathfrak{R}^{m \times m}$ and $\theta_{2}(t) \in \mathfrak{R}^{m \times m}$ are adaptive parameters updated according to (2.12).

For notation purposes, given a matrix $M$ we will denote $\operatorname{sgn}(M)$ as a matrix containing the sign of each element $m_{i j}$ of matrix $M$.

$b^{\prime}(y, z)$ is assumed to be invertible for all $x$, such that for the closed-loop system the following is satisfied:

$$
\begin{aligned}
\dot{V}= & \left(\frac{\partial V(y)}{\partial y}\right)^{T}\left\{A a^{\prime}(y, z)\right. \\
& +B b^{\prime}(y, z) b^{\prime}(y, z)^{-1}\left[\theta_{1}(t) a^{\prime}(y, z)\right. \\
& \left.\left.+\theta_{2}(t) u^{p}(t)\right]\right\}=y(t)^{T} u^{p}(t),
\end{aligned}
$$

where $V$ is a $C^{r}$-storage function and $u^{p}(t) \in \mathfrak{R}^{m}$ is the new control input.

The system made equivalent via the feedback according to Definition 2.7 is zero-state detectable according to Definition 2.3. Then, the control law

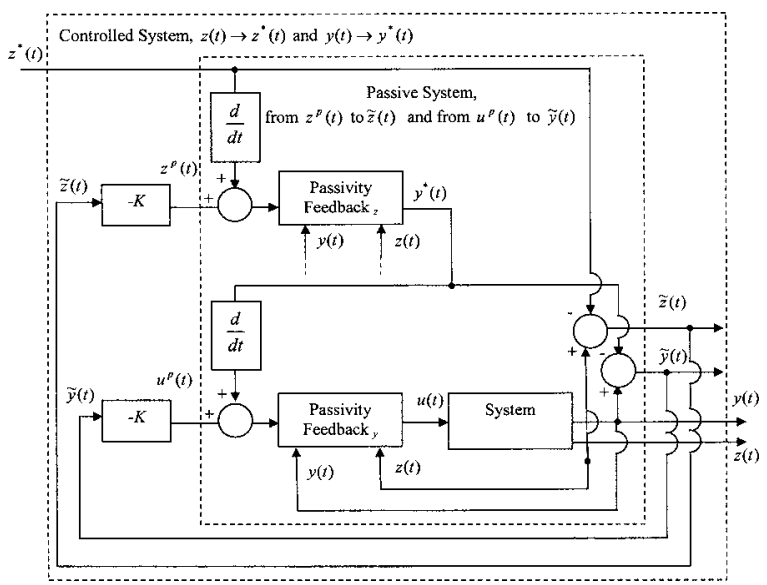

Fig. 2. General cascade PBC scheme.

given in (2.6) globally asymptotically stabilizes the system around the equilibrium point $x=0$.

\section{Cascade PBC for tracking purposes}

In this section a PBC scheme for cascade systems, which is valid for tracking control purposes, is shown. The scheme is shown in Fig. 2 and it is also valid for regulation and stabilization purposes. The general cascade PBC scheme is valid for nonadaptive and adaptive passivity feedback and will be discussed in Sections 3.1 and 3.2, respectively.

In the case of the tank truck mentioned in Section 2, the implementation of a speed controller could be designed for regulation purposes. The design of a route controller could also be used for tracking purposes.

The following assumptions are first introduced on the system to be considered.

Assumption 1: Let us assume that system (2.1) has relative degree $\{1,1,1, \ldots, 1\}$ at $x=0$, according to Definition 2.1.

Assumption 2: Let us assume that the distribution spanned by vector fields $g_{1}(x), \cdots, g_{m}(x)$ of system (2.1) is involutive [15].

Assumption 3: Let us assume that the inner loop [from $u^{p}(t)$ to $\widetilde{z}(t)$ ] in the scheme of Fig. 2 is faster (two or three times faster at least) than the outer loop [from $z^{p}(t)$ to $\left.\widetilde{z}(t)\right]$.

\subsection{Nonadaptive cascade PBC}

In this section we will present a $\mathrm{PBC}$ scheme for tracking purposes to control a cascade system of 
the form (2.3). It is important to note that we are not imposing that the system (2.3) be of minimum phase [that is to say that the zero dynamics $\dot{z}(t)$ $=f_{0}(C, z)$ be asymptotically stable].

Theorem 3.1: Let us consider the system (2.3) under the Assumptions 1 to 3. Let us also assume that $p(D, y, z) \in \mathfrak{R}^{m x m}$ is an invertible matrix for all $(y, z)$ around $(0,0)$. If we choose the following feedback:

$$
u(t)=b(B, y, z)^{-1} \theta_{y}^{T} \omega_{y}(t)
$$

with

$$
\begin{gathered}
\theta_{y}^{T}=\left[-I_{m} I_{m}\right] \in \mathfrak{R}^{m \times 2 m}, \\
\omega_{y}(t)=\left\{a^{T}(A, y, z)\left[u^{p T}(t)+\dot{y}^{* T}(t)\right]\right\}^{T} \in \mathfrak{R}^{2 m}
\end{gathered}
$$

and

with

$$
y^{*}(t)=p(D, y, z)^{-1} \theta_{z}^{T} \omega_{z}(t)
$$

$$
\begin{gathered}
\theta_{z}^{T}=\left[-I_{m} I_{m}\right] \in \mathfrak{R}^{m \times 2 m}, \\
\omega_{z}(t)=\left\{f_{0}^{T}(C, z)\left[z^{p T}(t)+z^{* T}(t)\right]\right\}^{T} \in \mathfrak{R}^{2 m},
\end{gathered}
$$

where $z^{*}(t)$ is any smooth desired trajectory, and $u^{p}(t)=-K_{y} \tilde{y}(t)$, with $K_{\mathrm{y}}$ $\in \mathfrak{R}^{m \times m}$ a positive definite matrix,

$z^{p}(t)=-K_{z} \tilde{z}(t)$, with $K_{\mathrm{z}}$

$$
\in \mathfrak{R}^{m \times n} \text { a positive definite matrix, }
$$

then the overall system is globally asymptotically stable around zero. That is to say $\lim _{t \rightarrow \infty} \widetilde{y}(t)$ $=\lim _{t \rightarrow \infty}\left[y(t)-y^{*}(t)\right]=0 \quad$ and $\quad \lim _{t \rightarrow \infty} \widetilde{z}(t)$ $=\lim _{t \rightarrow \infty}\left[z(t)-z^{*}(t)\right]=0$.

Proof 3.1: After applying Eqs. (3.1) and (3.3) to the system (2.3), the following system is obtained:

$$
\begin{gathered}
\dot{\tilde{y}}(t)=-K_{y} \tilde{y}(t), \\
\dot{z}(t)=f_{0}(C, z)+p(D, y, z) y(t),
\end{gathered}
$$

which guarantees that $y(t) \rightarrow y^{*}(t)$ even if $y^{*}$ is a time varying signal. Then $y(t)$ can track the trajectory defined by (3.2). Since the inner loop is faster than the outer loop, and after applying the controller defined by (3.2) and (3.4), we can write

$$
\begin{aligned}
& \dot{\tilde{y}}(t)=-K_{y} \tilde{y}(t), \\
& \dot{\tilde{z}}(t)=-K_{z} \widetilde{z}(t) .
\end{aligned}
$$

Equation (3.5) has associated positive definite storage functions $V_{y}=\frac{1}{2} \tilde{y}^{T}(t) \widetilde{y}(t)$ and $V_{z}$ $=\frac{1}{2} \widetilde{z}^{T}(t) \widetilde{z}(t)$, with $V_{y}(0)=0$ and $V_{z}(0)=0$, such that

$$
\begin{aligned}
& \dot{V}_{y}=\widetilde{y}^{T}(t) \dot{\tilde{y}}(t)=-\widetilde{y}^{T}(t) K_{y} \widetilde{y}(t), \\
& \dot{V}_{z}=\widetilde{z}^{T}(t) \dot{\tilde{z}}(t)=-\widetilde{z}^{T}(t) K_{z} \widetilde{z}(t) .
\end{aligned}
$$

Integrating both sides of the previous equations, we obtain

$$
\int_{0}^{\infty} \dot{V}_{y} d t=-\int_{0}^{\infty} \tilde{y}^{T}(t) K_{y} \tilde{y}(t) d t,
$$

$$
\int_{0}^{\infty} \dot{V}_{z} d t=-\int_{0}^{\infty} \widetilde{z}^{T}(t) K_{z} \widetilde{z}(t) d t
$$

which can be written as

$$
\begin{aligned}
& {\left[V_{y}(\infty)-V_{y}(0)\right]=-\int_{0}^{\infty} \tilde{y}^{T}(t) K_{y} \tilde{y}(t) d t,} \\
& {\left[V_{z}(\infty)-V_{z}(0)\right]=-\int_{0}^{\infty} \widetilde{z}^{T}(t) K_{z} \tilde{z}(t) d t .}
\end{aligned}
$$

Since $V_{y}, V_{z}$ are positive definite with negative definite derivatives $\dot{V}_{y}, \dot{V}_{z}$, the left-hand sides of (3.6) are finite, therefore $\tilde{y}(t) \in l^{2}, \widetilde{z}(t) \in l^{2}$. From system equations (2.3) we conclude that $\dot{\tilde{y}}(t) \in l^{\infty}$ and $\dot{\widetilde{z}}(t) \in l^{\infty}$. Using the Barbalat Lemma [10] we have that since $\tilde{y}(t) \in l^{2}, \widetilde{z}(t) \in l^{2}$ and $\dot{\tilde{y}}(t)$ $\in l^{\infty}, \dot{\tilde{z}}(t) \in l^{\infty}, \quad$ then $\lim \tilde{y}(t)=0$ and $\lim \tilde{z}(t)=0$.

Remark 3.1: Notice that after applying passivity feedbacks (3.1) and (3.2) to system (2.3) and considering $y(t)=y^{*}(t)$ as the input to $z(t)$ equation (since the inner loop is faster than the outer loop), we obtain

$$
\begin{aligned}
& \dot{\tilde{y}}(t)=u^{p}(t), \\
& \dot{\widetilde{z}}(t)=z^{p}(t),
\end{aligned}
$$

which is a passive equivalent system. 


\subsection{Adaptive cascade $P B C$}

We will now consider the case when the plant parameters of system (2.3) are unknown. We will consider the class of systems for which we assume that there exists a linear explicit parametric dependence of the form

$$
\begin{gathered}
\dot{y}(t)=A a^{\prime}(y, z)+B b^{\prime}(y, z) u(t), \\
\dot{z}(t)=C f_{0}^{\prime}(z)+D p^{\prime}(y, z) y(t),
\end{gathered}
$$

where $a \prime(y, z) \mathfrak{R}^{m}, A \in \mathfrak{R}^{m \times m}, b \prime(y, z) \in \mathfrak{R}^{m \times m}$, $B \in \mathfrak{R}^{m \times m}, \quad f_{0}^{\prime}(z) \in \mathfrak{R}^{n-m}, \quad C \in \mathfrak{R}^{(n-m) \times(n-m)}$, $p^{\prime}(y, z) \in \mathfrak{R}^{(n-m) \times(n-m)}, D \in \mathfrak{R}^{(n-m) \times(n-m)}$. It is assumed that $p^{\prime}(y, z) \in \mathfrak{R}^{(n-m) \times(n-m)}$ is an invertible matrix.

In what follows, to simplify the developments, it will be assumed that matrices $B$ and $D$ are diagonal. When $B$ and $D$ are positive definite matrices or more than that, they are just general matrices and can be studied using the same ideas presented in $[6,7]$. So in this paper only the case of diagonal matrices will be considered.

Again it is important to note that it is not assumed that the system (2.3) is of minimum phase. We can now state a similar theorem to Theorem 3.1 but for the adaptive case.

Theorem 3.2: Let us consider the system of the form (3.7) under Assumptions 1 to 3, with $p^{\prime}(y, z)$ an invertible matrix for all $(y, z)$ around $(0,0)$. If we choose the following feedback

$$
u(t)=b^{\prime}(y, z)^{-1} \theta_{y}(t)^{T} \omega_{y}(t)
$$

with

$$
\begin{gathered}
\theta_{y}^{T}(t)=\left[\theta_{y 1}(t) \theta_{y 2}(t)\right] \in \mathfrak{R}^{m \times 2 m}, \\
\omega_{y}(t)=\left\{a^{\prime T}(y, z)\left[u^{p T}(t)+\dot{y}^{* T}(t)\right]\right\}^{T} \in \mathfrak{R}^{2 m}
\end{gathered}
$$

and

$$
y^{*}(t)=p^{\prime}(y, z)^{-1} \theta_{z}(t)^{T} \omega_{z}(t)
$$

with

$$
\begin{gathered}
\theta_{z}^{T}(t)=\left[\theta_{z 1}(t) \theta_{z 2}(t)\right] \in \mathfrak{R}^{n-m \times 2(n-m)}, \\
\omega_{z}(t)=\left\{f_{0}^{\prime T}(z)\left[z^{p T}(t)+\dot{z}^{* T}(t)\right]\right\}^{T} \in \mathfrak{R}^{2(n-m)}
\end{gathered}
$$

with adaptive laws defined as

$$
\dot{\theta}_{y}(t)^{T}=-\operatorname{sgn}(B) \tilde{y}(t) \omega_{y}(t)^{T},
$$

$$
\dot{\theta}_{z}(t)^{T}=-\operatorname{sgn}(D) \widetilde{z}(t) \omega_{z}(t)^{T},
$$

and

$$
\begin{aligned}
u^{p}(t)= & -K_{y} \widetilde{y}(t), \text { with } K_{\mathrm{y}} \\
& \in \Re^{m \times m} \text { a positive definite matrix, } \\
z^{p}(t)=- & K_{z} \widetilde{z}(t), \text { with } K_{\mathrm{z}} \\
& \in \mathfrak{R}^{m \times m} \text { a positive definite matrix, }
\end{aligned}
$$

then the overall adaptive system is globally uniformly bounded and $\lim _{t \rightarrow \infty} \widetilde{y}(t)=\lim _{t \rightarrow \infty}[y(t)$ $\left.-y^{*}(t)\right]=0$ and $\lim _{t \rightarrow \infty} \tilde{z}(t)=\lim _{t \rightarrow \infty}\left[z(t)-z^{*}(t)\right]$ $=0$.

Proof 3.2. After applying Eqs. (3.8), (3.10), and (3.11), to the system (3.7), the following system is obtained:

$$
\begin{gathered}
\dot{\tilde{y}}(t)=-K_{y} \tilde{y}(t)+\phi_{y}(t)^{T} \omega_{y}, \\
\dot{z}(t)=C f_{0}^{\prime}(z)+D p^{\prime}(y, z) y(t),
\end{gathered}
$$

where $\quad \phi_{y}(t)=\theta_{y}(t)-\theta_{y}^{*} \in \mathfrak{R}^{m \times 2 m}$, and $\theta_{y}^{*}$ $=\left[-B^{-1} A B^{-1}\right]^{T} \in \mathfrak{R}^{m \times 2 m}$ are the ideal parameters of the passivator. This guarantees that $y(t)$ $\rightarrow y^{*}(t)$ even if $y(t)^{*}$ is a time varying signal. Then $y(t)$ can track the trajectory defined by (3.9) and (3.11). Finally, after applying the outer controller defined by (3.9), (3.11), and (3.13) and considering that the inner loop is faster than the outer loop, we can write

$$
\begin{gathered}
\dot{\tilde{y}}(t)=-K_{y} \tilde{y}(t)+B \phi_{y}(t)^{T} \omega_{y}, \\
\dot{\widetilde{z}}(t)=-K_{z} \widetilde{z}(t)+D \phi_{z}(t)^{T} \omega_{z},
\end{gathered}
$$

where $\phi_{z}(t)=\theta_{z}(t)-\theta_{z}^{*} \in \mathfrak{R}^{n-m \times 2(n-m)}$, and $\theta_{y}^{*}$ $=\left[-D^{-1} C D^{-1}\right]^{T} \in \Re^{n-m \times 2(n-m)}$ are the ideal parameters of the passivator.

For notation purposes, given a matrix $M$ we will denote $\operatorname{abs}(M)$ as a matrix containing the absolute value of each element $m_{i j}$ of matrix $M$.

Eq. (3.14) has associated positive definite storage functions $V_{y}=\frac{1}{2} \widetilde{y}^{T}(t) \widetilde{y}(t)$ $+\frac{1}{2} \operatorname{tr}\left[\operatorname{abs}\left(B^{T}\right) \phi_{y}^{T}(t) \phi_{y}(t)\right] \quad$ and $\quad V_{z}=\frac{1}{2} \widetilde{z}^{T}(t) \widetilde{z}(t)$ $+\frac{1}{2} \operatorname{tr}\left[\operatorname{abs}\left(D^{T}\right) \phi_{z}^{T}(t) \phi_{z}(t)\right]$, with $\quad V_{y}(0)=0 \quad$ and $V_{z}(0)=0$, such that 


$$
\begin{aligned}
& \dot{V}_{y}(y)=\widetilde{y}(t)^{T} \dot{\tilde{y}}(t)+\operatorname{tr}\left[\operatorname{abs}\left(B^{T}\right) \dot{\phi}_{y}^{T}(t) \phi_{y}(t)\right], \\
& \dot{V}_{z}(z)=\widetilde{z}(t)^{T} \dot{\widetilde{z}}(t)+\operatorname{tr}\left[\operatorname{abs}\left(D^{T}\right) \dot{\phi}_{z}^{T}(t) \phi_{z}(t)\right] .
\end{aligned}
$$

Substituting (3.14) in (3.15), considering the vector property $a^{T} b=b^{T} a=\operatorname{tr}\left(b a^{T}\right)=\operatorname{tr}\left(a b^{T}\right)$ and regrouping terms, we have

$$
\begin{aligned}
\dot{V}_{y}(y)= & -\widetilde{y}(t)^{T} K_{y} \widetilde{y}(t)+\operatorname{tr}\left[B^{T} \widetilde{y}(t) \omega_{y}^{T} \phi_{y}(t)\right. \\
& \left.+\operatorname{abs}\left(B^{T}\right) \dot{\phi}_{y}^{T}(t) \phi_{y}(t)\right], \\
\dot{V}_{z}(z)= & -\widetilde{z}(t)^{T} K_{z} \widetilde{z}(t)+\operatorname{tr}\left[D^{T} \widetilde{z}(t) \omega_{z}^{T} \phi_{z}(t)\right. \\
& \left.+\operatorname{abs}\left(D^{T}\right) \dot{\phi}_{z}^{T}(t) \phi_{z}(t)\right] .
\end{aligned}
$$

Considering $\dot{\phi}_{y}^{T}(t)=\dot{\theta}_{y}^{T}(t), \dot{\phi}_{z}^{T}(t)=\dot{\theta}_{z}^{T}(t)$, substituting the adaptive laws defined by Eqs. (3.10) and (3.11) but reexpressing them as $\dot{\theta}_{y}(t)^{T}$ $=-\operatorname{sgn}(B) \tilde{y}(t) \omega_{y}(t)^{T} \quad$ and $\quad \dot{\theta}_{z}(t)^{T}=$ $-\operatorname{sgn}(D) \widetilde{z}(t) \omega_{z}(t)^{T}$, respectively, and knowing that $B^{T}=B=\operatorname{abs}(B) \operatorname{sgn}(B)$ and $D^{T}=D=\operatorname{abs}(D) \operatorname{sgn}(D)$, we finally obtain

$$
\begin{aligned}
& \dot{V}_{y}=-\widetilde{y}^{T}(t) K_{y} \widetilde{y}(t), \\
& \dot{V}_{z}=-\widetilde{z}^{T}(t) K_{z} \widetilde{z}(t) .
\end{aligned}
$$

Integrating both sides of the previous equations, we get

$$
\begin{aligned}
& \int_{0}^{\infty} \dot{V}_{y} d t=-\int_{0}^{\infty} \tilde{y}^{T}(t) K_{y} \widetilde{y}(t) d t, \\
& \int_{0}^{\infty} \dot{V}_{z} d t=-\int_{0}^{\infty} \widetilde{z}^{T}(t) K_{z} \widetilde{z}(t) d t,
\end{aligned}
$$

which can be expressed as

$$
\begin{aligned}
& {\left[V_{y}(\infty)-V_{y}(0)\right]=-\int_{0}^{\infty} \widetilde{y}^{T}(t) K_{y} \widetilde{y}(t) d t,} \\
& {\left[V_{z}(\infty)-V_{z}(0)\right]=-\int_{0}^{\infty} \widetilde{z}^{T}(t) K_{z} \widetilde{z}(t) d t .}
\end{aligned}
$$

Since $V_{y}, V_{z}$ are positive definite and its derivatives $\dot{V}_{y}, \dot{V}_{z}$ are negative definite, the left-hand sides of (3.16) are finite, therefore $\widetilde{y}(t) \in l^{2}, \widetilde{z}(t)$ $\in l^{2}$. From the system Eqs. (3.6) we can conclude
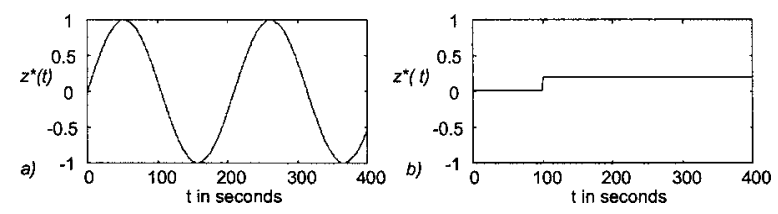

Fig. 3. Reference signals considered in the simulations. (a) Sine wave of amplitude 1 and frequency $0.03 \mathrm{rad} / \mathrm{s}$. (b) Step of amplitude 0.1 at $t=100$.

that $\dot{\tilde{y}}(t) \in l^{\infty}, \dot{\tilde{z}}(t) \in l^{\infty}$. Using the Barbalat Lemma $[10], \quad \tilde{y}(t) \in l^{2}, \quad \widetilde{z}(t) \in l^{2} \quad$ and $\quad \dot{\tilde{y}}(t) \in l^{\infty}, \quad \dot{\tilde{z}}(t)$ $\in l^{\infty} \Rightarrow \lim _{t \rightarrow \infty} \widetilde{y}(t)=0, \lim _{t \rightarrow \infty} \tilde{z}(t)=0 . \diamond$

Remark 3.2: Notice that after applying the passivity feedbacks (3.8) and (3.9) to system (3.7), and considering $y(t)=y^{*}(t)$ as the input to equation $z(t)$ (since the inner loop is faster than the outer loop), it is obtained

$$
\begin{aligned}
& \dot{\tilde{y}}(t)=u^{p}(t)+\phi_{y}^{T}(t) \omega_{y}(t), \\
& \dot{\widetilde{z}}(t)=z^{p}(t)+\phi_{z}^{T}(t) \omega_{z}(t),
\end{aligned}
$$

which is a passive equivalent system.

\section{Simulation Results}

In this section we simulate the proposed control methodology considering the case when the plant parameters are known and then we also study the adaptive case.

Two different reference signals for $z^{*}(t)$ will be used in the simulations, as shown in Fig. 3. The first reference is used to study the behavior of the system under tracking and the second one is for regulation purposes.

\subsection{Example 4.1: The nonadaptive case}

Let us control a system of the form (2.1) with

$$
f(x)=\left[\begin{array}{c}
-3 x_{1}^{2}-x_{2} \\
x_{1}-5 x_{2}^{3}
\end{array}\right], \quad g(x)=\left[\begin{array}{l}
1 \\
0
\end{array}\right], \quad h(x)=x_{1},
$$

where $x(t)=\left[\begin{array}{ll}x_{1} & x_{2}\end{array}\right]^{T} \in \mathfrak{R}^{2}$ and $y(t), u(t) \in \mathfrak{R}$ are scalars. Here we have $n=2 m=2$.

Now we will check the Assumptions 1 and 2 for system (4.1). This system has zero equilibrium point $(0,0), \quad$ relative degree $L_{g} h(x)$ $=[\partial h(x) / \partial x] g(x)=1 \neq 0$, and the distribution 

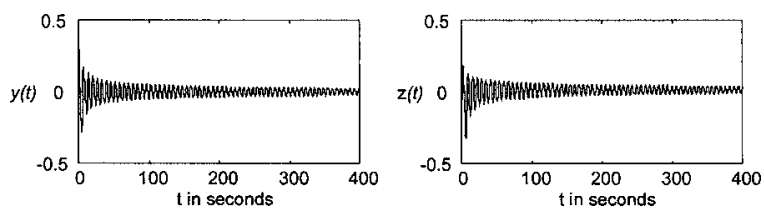

Fig. 4. Natural behavior of the system of Example 4.1.

spanned by $g(x)=\left[\begin{array}{ll}1 & 0\end{array}\right]^{T}$ is involutive. The system can then be represented in the form (2.3) by considering

$$
\begin{gathered}
y(t)=x_{1}(t), \quad z(t)=x_{2}(t), \\
a(y, z)=-\left(3 y^{2}+z\right), \quad b(y, z)=1, \\
f_{0}(z)=-5 z^{3}, \quad p(y, z)=1 .
\end{gathered}
$$

It can be easily checked that system (4.2) is weekly minimum phase according to Definition 2.2. Also, it has associated a positive storage function $W_{0}=\frac{1}{2} z^{2}$ with $W_{0}(0)=0$, such that $L_{c(0, z)} W_{0}(z)=\left[\partial W_{0}(x) / \partial z\right] c(0, z)=-5 z^{4} \leqslant 0$.

Let us first study the natural behavior of the system of Example 4.1. The unforced response of (4.2) with $y(0)=0.5, z(0)=-0.5$ is shown in Fig. 4.

It can be seen that the system exhibits oscillations and a stable $z(t)$ state, so it is verified that the system is weekly minimum phase.

We now consider the nonadaptive cascade PBC scheme presented in Section 3.1, defined by the control law of the form (3.1)-(3.4) with

$$
\begin{gathered}
\omega_{y}(t)=\left\{-\left(3 y^{2}+z\right)\left[u^{p}(t)+\dot{y}^{*}(t)\right]\right\}^{T} \in \mathfrak{R}^{2}, \\
\omega_{z}(t)=\left\{-5 z^{3}\left[z^{p}(t)+\dot{z}^{*}(t)\right]\right\}^{T} \in \mathfrak{R}^{2},
\end{gathered}
$$

which applied to system (4.2) will guarantee that $y(t) \rightarrow y^{*}(t)$ and $z(t) \rightarrow z^{*}(t)$ asymptotically, regardless of the initial conditions. The control scheme for this example is shown in Fig. 5.

Fig. 6 shows the behavior of the controlled system of Example 4.1, with $K_{y}=-40, K_{z}=-1$ and when a sinusoidal reference signal of form shown in Fig. 3(a) is applied for $z^{*}(t)$.

From Figs. 6(e) and 6(f) it can be seen that $\tilde{y}(t) \rightarrow 0$ and $\widetilde{z}(t) \rightarrow 0$. A similar result is shown in Fig. 7 but when a step reference signal at $t=100$ is applied for $z^{*}(t)$. [See Fig. 3(b)].

It is important to notice in Fig. 7 that when applying a step reference, its derivative tends to in-

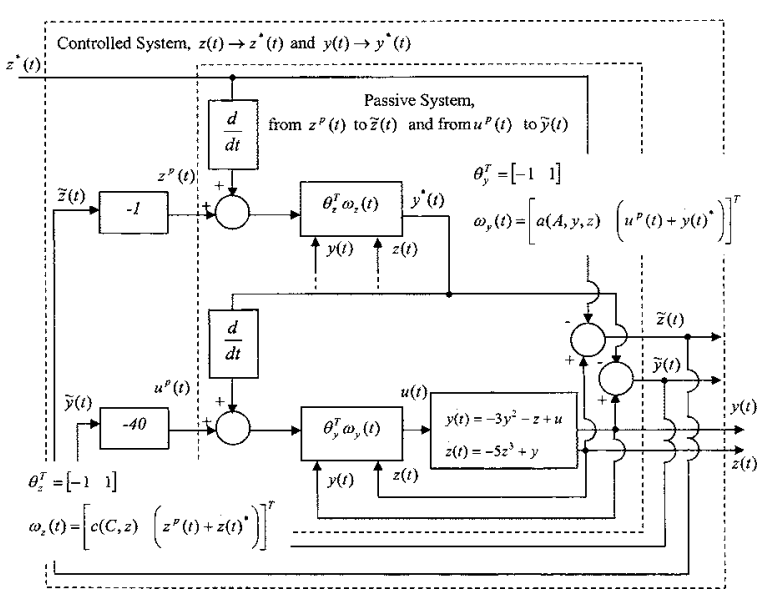

Fig. 5. Control scheme for Example 4.1. Nonadaptive case.

finite at $t=100$. So for this case a practical solution shall be applied, as, for example, the use of saturation blocks.

\subsection{Example 4.2. The adaptive case}

Let us control the system of Example 4.1 but assuming now that the plant parameters are unknown. The system can be expressed in the form of (3.7), where for this particular example we have

$$
\begin{gathered}
y(t)=x_{1}, \quad z(t)=x_{2}, \\
A a^{\prime}(y, z)=\left[\begin{array}{ll}
-3 & -1
\end{array}\right]\left[\begin{array}{c}
y^{2} \\
z
\end{array}\right],
\end{gathered}
$$
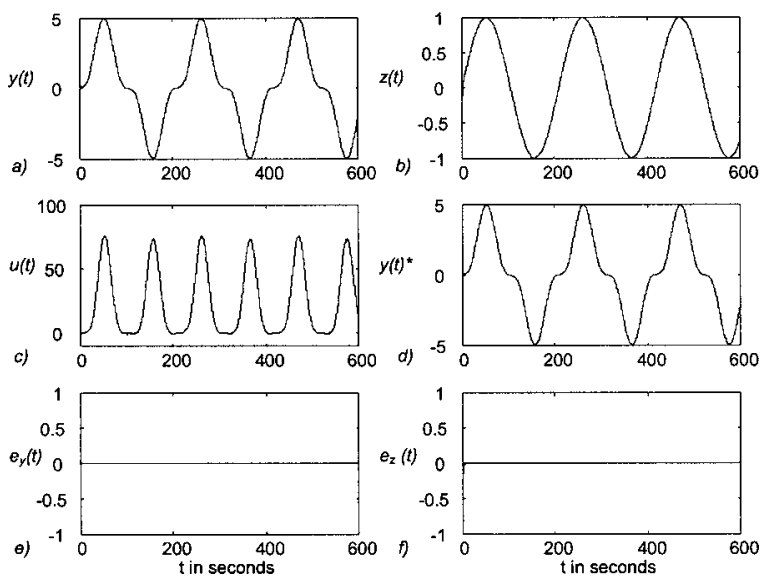

Fig. 6. Simulation results for the control scheme of Example 4.1. Tracking control. 

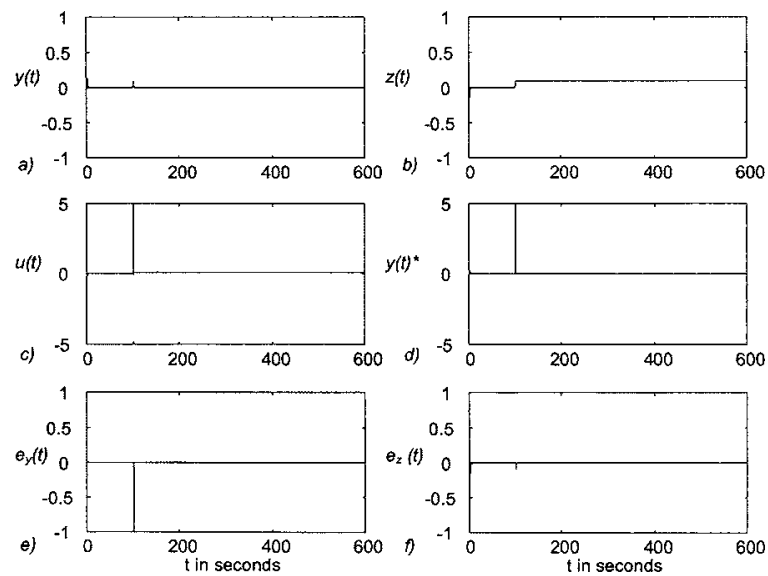

Fig. 7. Simulation result for the control scheme of Example 4.1. Regulation control.

$$
B b^{\prime}(y, z)=1, \quad C c^{\prime}(z)=(-5) z^{3}, \quad p^{\prime}(y, z)=1 .
$$

Since the parameters $A, B, C$, and $D$ are assumed to be unknown, we will use the adaptive cascade PBC scheme discussed in Section 3.2. After applying to the system (4.4) a control law of the form (3.8), (3.9), (3.12), and (3.13) with

$$
\begin{gathered}
\omega_{y}(t)=\left\{y^{2} z\left[u^{p}(t)+\dot{y}(t)^{*}\right]\right\}^{T} \in \mathfrak{R}^{2}, \\
\omega_{z}(t)=\left\{z^{3}\left[z^{p}(t)+\dot{z}(t)^{*}\right]\right\}^{T} \in \mathfrak{R}^{2}
\end{gathered}
$$

and the adaptive laws defined in (3.10) and (3.11) it is guaranteed that the state will follow the desired trajectories defined by $z^{*}(t)$ and $y^{*}(t)$. The control scheme is shown in Fig. 8.

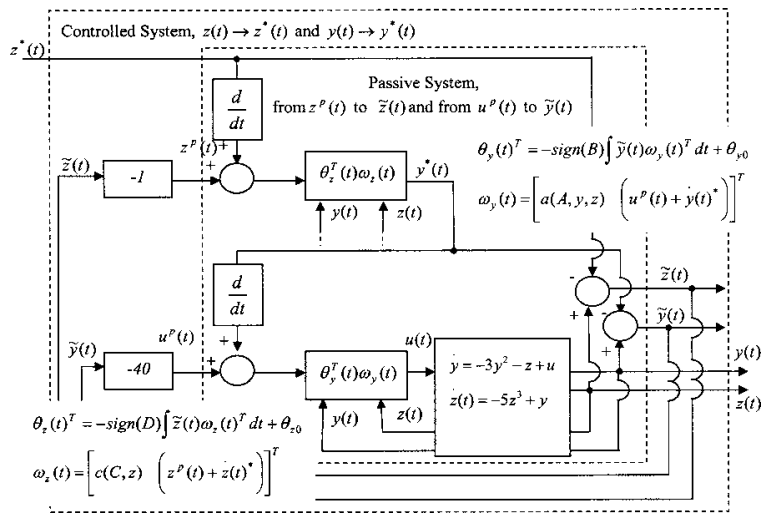

Fig. 8. Control scheme for Example 4.2. Adaptive case.
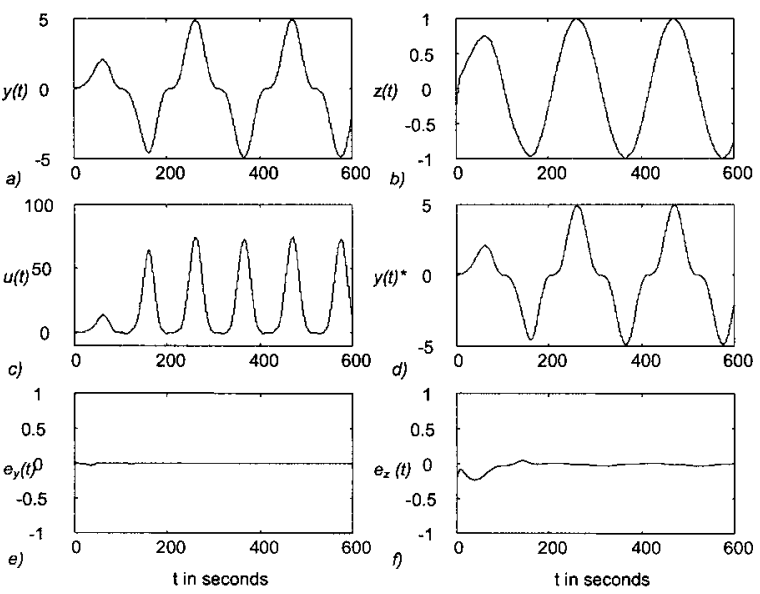

Fig. 9. Simulation result for the control scheme of Example 4.2. Tracking control.

Fig. 9 shows the simulation results when a sinusoidal reference signal of the form shown in Fig. 3(a) is applied to $z^{*}(t)$, and the feedback gains are $K_{y}=-40, K_{z}=-1$.

Similar to the nonadaptive case, the tracking errors go to zero asymptotically, as can be seen from Figs. 9(e) and 9(f). In Fig. 10, we can see the evolution of the controller parameters.

Similar results can be found in Figs. 11 and 12 but for the case when a step reference signal is used for $z^{*}(t)$. Again it can be observed that the tracking errors go to zero asymptotically.

The simulation example used in this section has no direct industrial counterpart, and it was chosen because it contains severe nonlinearities (quadratic and cubic type). The idea was to show that the proposed control system behaves well even in this adverse case. In other industrial cases with mild nonlinearities (like the model of an induction motor where we have products between state variables), we expect a good behavior as well.

\section{Conclusions}

A new PBC scheme for cascade nonlinear systems, applicable for tracking, regulation, and sta-
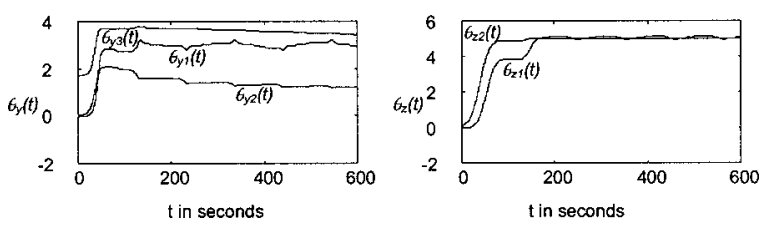

Fig. 10. Controller parameters of Example 4.2. Tracking control. 

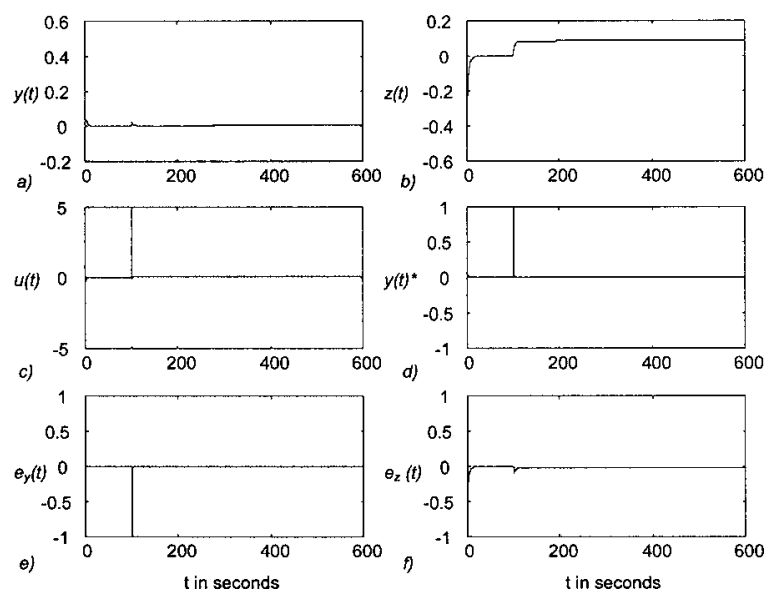

Fig. 11. Simulation result for the control scheme of Example 4.2. Regulation control.

bilization, has been proposed in this paper. It can be applied to the control of cascade systems that are not necessarily weakly minimum phase. The methodology can be applied for the case when all system parameters are assumed to be known (nonadaptive case), and also, with introduction of appropriate adaptive laws, it can be used in the adaptive case, provided the plant can be parametrized in such a fashion that the unknown parameters enter linearly in the equations. Although in the latter the development presented in the paper was done only for the case when the matrices $B$ and $D$ are diagonal, the extension to the case when those matrices are positive definite or even just general matrices can be performed using the same ideas given in $[6,7]$.

The simulation examples given for the nonadaptive and adaptive case in Section 4 reveal that the behavior of the proposed control scheme is in complete agreement with the theoretical results presented in Section 3.

\section{Acknowledgments}

The results reported in this paper have been supported by CONICYT-CHILE through FOND-

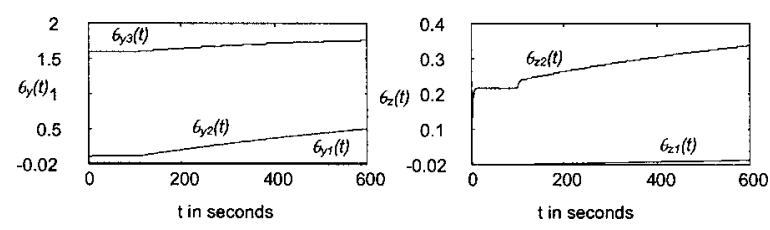

Fig. 12. Controller parameters of Example 4.2. Regulation control.
ECYT Grant No. 1030962.

\section{References}

[1] Desoer, C. A. and Vidyasagar, M., Feedback Systems: Input-output Properties. Academic Press, New York, 1975.

[2] Hill, D. and Moylan, P., Stability results for nonlinear feedback systems. Automatica 13, 373-382 (1977).

[3] Willems, J. C., Dissipative dynamical systems. Part I: General theory. Arch. Ration. Mech. Anal. 45, 325351 (1972).

[4] Willems, J. C., Dissipative dynamical systems. Part II: Linear systems with quadratic suply rates. Arch. Ration. Mech. Anal. 45, 352-393 (1972).

[5] Byrnes, C. I., Isidori, A., and Willems, J. C., Passivity, feedback equivalence, and the global stabilization of minimun phase nonlinear systems. IEEE Trans. Autom. Control 36, 1228-1240 (1991).

[6] Duarte-Mermoud, M. A., Méndez-Miquel, J. M., Castro-Linares, R., and Castillo-Facuse, A., Adaptive passivation with time-varying gains of MIMO nonlinear systems. Kybernetes 32(9/10), 1342-1368 (2003).

[7] Duarte-Mermoud, M. A., Castro-Linares, R., and Castillo-Facuse, A., Direct passivity of a class of MIMO nonlinear systems using adaptive feedback. Int. J. Control 75(1), 23-33 (2002).

[8] Duarte-Mermoud, M. A., Castro-Linares, R., and Castillo-Facuse, A., Adaptive passivity of nonlinear systems using time-varying gains. Dyn. Control 11(4), 333-351 (2001).

[9] Castro-Linares, R. and Duarte-Mermoud, M. A., Passivity equivalence of a class of nonlinear systems via adaptive feedback. Proceedings of the 8th Latin American Congress on Automatic Control, November 9-13, 1998, Marbella, Chile, ACCA, pp. 249-254.

[10] Narendra, K. S. and Annaswamy, A. M., Stable Adaptive Systems. Prentice-Hall, Inc., Englewood Cliffs, NJ, 1988.

[11] Travieso-Torres, J. C. and Duarte-Mermoud, M. A., Equivalence between adaptive passivity based control and model reference adaptive control. WSEAS Trans. Circuit Syst. 4(9), 1912-1917 (2004).

[12] Travieso-Torres, J. C. and Duarte-Mermoud, M. A., Passivity-based control for stabilization, regulation and tracking purposes of a class of nonlinear systems. Submitted to the Int. J. Adapt. Control Signal Process.

[13] Ortega, R., Passivity properties for stabilization of cascade nonlinear systems. Automatica 27(2), 423-424 (1991).

[14] Huang, S, James, M. R., and Jiang, Z. P., L ${ }^{\infty}$-bounded robust control of nonlinear cascade systems. Syst. Control Lett. 54(3), 215-224 (2005).

[15] Khalil, H. K., Non Linear Systems. 3rd ed., Prentice Hall, Englewood Cliffs, NJ, 2003.

[16] Ortega, R. and Espinoza-Pérez, G., Passivity based control with simultaneous energy-shaping and damping injection: The induction motor case. Proceedings of the 16th IFAC World Congress, July 3-8, 2005, Prague, Czech Republic. Proceedings in CD, Track We-E20-TO/3, 6 pp.

[17] Salazar, R., Rodríguez, P., Duarte-Mermoud, M. A., 
Travieso, J. C., La Rosa, P. S., Bustos, M. A., and González, R. I., Control of a levitation system by adaptive feedback passivity. Proceedings of the 10th Latin American Congress on Automatic Control, Guadalajara, Mexico, December 3-6, 2002. Proceedings in CD, Track No. 156, 6 pp.

[18] Duarte, M., Castro, R., and Castillo, A., Adaptive passivation of a magnetic levitator system using timevarying gains. Proceedings of the First IFAC/IEEE Symposium on System Structure and Control, edited by P. Horacek, Prague, Czech Republic, August 29-31 2001. Proceedings in CD, Track No. 040, 6 pp.

[19] Duarte-Mermoud, M. A. and Travieso-Torres, J. C. Control of induction motors: An adaptive passivity MIMO perspective. Int. J. Adapt. Control Signal Process. 17(4), 313-332 (2003).

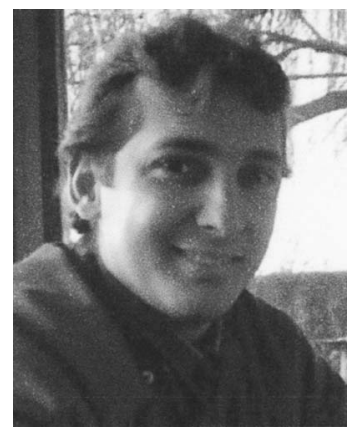

Juan C. Travieso-Torres Dr. Travieso-Torres received the degrees of Electrical Engineer and M.Sc. from the Superior Polytechnic Institute "José Antonio Echeverría" at Havana, Cuba, in 1995 and 2000, respectively; and the Ph.D. degree at the University of Santiago of Chile in 2003. He has ten years of professional experience working for different companies in Cuba and Chile, and six years of teaching experience at the Electrical Engineering Departments of the University of Santiago of Chile, and at the University of Chile. Since 2003 he has also worked at Fluor Corporation in Chile, where he is considered a global expert in the subjects of Digital Control Systems (DCS/PLC), Process Control (Control Strategies), and Variable Speed Drives, which are his main research areas.

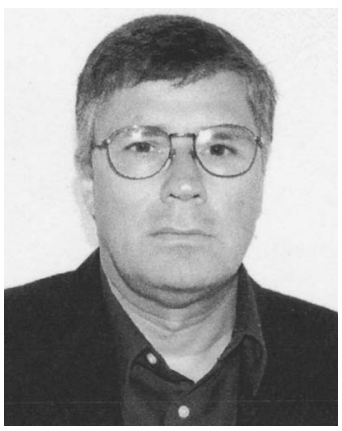

Manuel A. Duarte-Mermoud Dr. Duarte-Mermoud received the degree of Civil Electrical Engineer from the University of Chile in 1977 and the M.Sc., M.Phil. and the Ph.D. degrees, all in electrical engineering, from Yale University in 1985, 1986, and 1988, respectively. From 1977 to 1979 , he worked as Field Engineer at Santiago Subway. In 1979, he joined the Electrical Engineering Department of the University of Chile, where he is currently Professor. His main research interests are in robust adaptive control (linear and nonlinear systems) and system identification. Dr. Duarte is a member of the IEEE and IFAC. He is past Treasurer and past President of ACCA, the Chilean National Member Organization of IFAC, and past Vice-President of the IEEE-Chile.

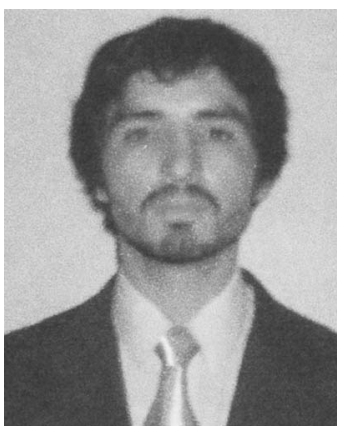

Jorge L. Estrada Mr. Estrada received the degrees of Electrical Engineer and M.Sc. in 2004 from the Electrical Engineering Department of the University of Chile in Santiago, Chile. He is currently working for the company IDT S.A., as control engineer in different manufacturing projects. His main research areas are nonlinear control and modeling. 